Originalien

Z Gerontol Geriat 2020 · 53:239-244 https://doi.org/10.1007/s00391-020-01712-7 Eingegangen: 16. Januar 2020

Angenommen: 10. Februar 2020

Online publiziert: 2. März 2020

(c) Der/die Autor(en) 2020

\author{
Martin Jäger ${ }^{1}$ Ulrich Thiem ${ }^{2,3} \cdot$ Holger Stege $^{4}$ \\ ${ }^{1}$ Hüttenhospital gemeinnützige GmbH, Dortmund, Deutschland \\ ${ }^{2}$ Medizinisch-Geriatrische Klinik, Albertinen-Haus Hamburg, Hamburg, Deutschland \\ ${ }^{3}$ Lehrstuhl für Geriatrie und Gerontologie, Universitätsklinik Eppendorf (UKE), Hamburg, Deutschland \\ ${ }^{4}$ Klinik für Geriatrie, Ruppiner Kliniken $\mathrm{GmbH}$, Hochschulklinikum der Medizinischen Hochschule \\ Brandenburg (MHB), Neuruppin, Deutschland
}

\title{
Entwicklung eines neuen Screeninginstruments zum Screening auf Dysphagie bei geriatrischen Patienten: das Dysphagie Screening-Tool Geriatrie
}

\section{Hintergrund}

Die oropharyngeale Dysphagie ist definiert als eine Funktionsstörung des Schluckakts bzw. eine Störung von Nahrungs- und Flüssigkeitsaufnahme sowie -transport. Diese allgemein gefasste Definition der Dysphagie erfordert keine bewusste Wahrnehmung des Betroffenen und umfasst damit auch das Phänomen der stillen Aspiration [13, 30].

Die Abgrenzung von alterstypischen Veränderungen $\mathrm{zu}$ pathologischen $\mathrm{Be}$ funden bereitet v.a. im Zusammenhang mit der Presbyphagie Probleme. Einerseits kann es zu komplexen Veränderungen des Schluckakts durch physiologische Alterungsprozesse mit Abnahme der Muskelmasse (Sarkopenie), Abnahme der Elastizität des Bindegewebes, Skelettveränderungen, Veränderungen der Sensorik und Sensibilität sowie der Modifikation der zentralen Steuerung des Schluckakts kommen (primäre Presbyphagie). Der primären Presbyphagie mit daraus resultierender leichter Dysphagie kommt oft kein Krankheitswert zu. Allerdings erhöht sich durch abnehmende Kompensationsreserve die Anfälligkeit für eine schwerere Dysphagie im Fall einer begleitenden Erkrankung. Ande- rerseits hat die sekundäre Presbyphagie - d.h. Presbyphagie als direkte Folge einer Erkrankung im höheren Lebensalter - große Relevanz [18]. Die Bedeutung und die Häufigkeit dieser Veränderungen führen zur Einordnung der oropharyngealen Dysphagie als geriatrisches Syndrom [2].

Die oropharyngeale Dysphagie ist im höheren Lebensalter ein Prädiktor für schwerwiegende Komplikationen und erhöhte Mortalität [18]. Bei klinischer Beurteilung der oropharyngealen Dysphagie kommt es zu Fehleinschätzungen, da sich die Symptome meist untypisch und vieldeutig darstellen oder z. B. durch eine Störung der pharyngealen und laryngealen Sensibilität ganz ausbleiben können [23]. Besondere Schwierigkeiten bereitet das oft fehlende Problembewusstsein der Betroffenen [4, 23]. Häufig wird eine Schluckstörung als Ausdruck des physiologischen Alterungsprozesses angesehen, sodass keine Bemühungen zu Diagnostik und Behandlung folgen [29]. Etwa 40-60\% der Schluckstörungen werden klinisch nicht erkannt $[1,3$, $9,16,19]$.

Ein standardisiertes Dysphagiemanagement bewirkt eine Senkung von Pneumonie- und Mortalitätsrate mit re- lativer Risikoreduktion von etwa 70-80\% und kann zudem zu einer relevanten Kostenreduktion führen $[9,12,16,19,22,24]$. Trotzdem findet in vielen Einrichtungen kein standardisiertes Dysphagiescreening oder -management statt $[9,19]$.

\section{Gängige Instrumente zum Dysphagiescreening}

Das Ziel von Screeninginstrumenten ist die frühzeitige Identifikation von Risikopatienten. Die Mehrzahl der Risikopatienten sollte auf der Ebene der Grundversorgung durch geschulte Mitarbeiter im Gesundheitssystem entdeckt werden. Zum Dysphagiescreening wurden verschiedene Verfahren v. a. bei Schlaganfallpatienten validiert ( $\bullet$ Tab. 1). Perry und Love beschrieben 2001 eine durchschnittliche Sensitivität der gängigen Screeningverfahren zur Detektion eines Aspirationsrisikos $>70 \%$ (Spezifität von 69-92 \%) [20]. Ramsey et al. wiesen 2003 auf eine Sensitivität von 42 bis $97 \%$ bei einer Spezifität von 59 bis $91 \%$ hin [21]. Das Scottish Intercollegiate Guidelines Network (SIGN) empfiehlt 2010 einen Wasserschlucktest, der Elemente verschiedener Screeningtests kombiniert, wie z.B. 2 von 6 Elementen nach Da- 


\begin{tabular}{|c|c|c|c|c|c|c|c|c|}
\hline Erstautor & Jahr & Instrument & Beschreibung & Fallzahl & Sens & Spez & PPV & NPV \\
\hline $\begin{array}{l}\text { Smithard } \\
\text { [26] }\end{array}$ & 1998 & BSA & $\begin{array}{l}\text { P: Schlaganfall, Akutphase } \\
\text { G: Videofluoroskopie } \\
\text { E: Aspirationspneumonie }\end{array}$ & 83 & 47 & 86 & 50 & 85 \\
\hline $\begin{array}{l}\text { De Pippo } \\
\text { [8] }\end{array}$ & 1992 & BDST & $\begin{array}{l}\text { P: Schlaganfall, Rehabilitation } \\
\text { G: Videofluoroskopie } \\
\text { E: Pneumonie, Asphyxie, Tod }\end{array}$ & 44 & 76 & 59 & - & - \\
\hline $\begin{array}{l}\text { Gottlieb } \\
\text { [11] }\end{array}$ & 1996 & - & $\begin{array}{l}\text { P: Schlaganfall, Rehabilitation } \\
\text { G: kein, klin. Assessment nach Logemann } \\
\text { E: oropharyngeale Dysphagie, Pneumonie }\end{array}$ & 180 & 85 & 45 & - & - \\
\hline $\begin{array}{l}\text { Splaingard } \\
\text { [27] }\end{array}$ & 1988 & - & $\begin{array}{l}\text { P: Schlaganfall, Rehabilitation } \\
\text { G: Videofluoroskopie } \\
\text { E: Aspirationsrisiko }\end{array}$ & 137 & 42 & 91 & 75 & 70 \\
\hline Daniels [6] & 1997 & - & $\begin{array}{l}\text { P: Schlaganfall, Akutphase } \\
\text { G: Videofluoroskopie } \\
\text { E: oropharyngeale Dysphagie, ab mittelschwer }\end{array}$ & 59 & 92 & 67 & - & - \\
\hline Trapl [28] & 2007 & GUSS & $\begin{array}{l}\text { P: Schlaganfall, Akutphase } \\
\text { G: FEES } \\
\text { E: Aspirationsrisiko }\end{array}$ & 50 & 100 & 50 & 81 & 100 \\
\hline $\begin{array}{l}\text { Martino } \\
\text { [17] }\end{array}$ & 2009 & TOR-BSST & $\begin{array}{l}\text { P: Schlaganfall, Akut- und Rehabilitationsphase } \\
\text { G: Videofluoroskopie } \\
\text { E: oropharyngeale Dysphagie }\end{array}$ & 311 & 91 & 67 & 93 & 46 \\
\hline SIGN [1] & 2010 & $\begin{array}{l}\text { Schottischer } \\
\text { Wasserschluck- } \\
\text { test }\end{array}$ & $\begin{array}{l}\text { P: Schlaganfall, Akut- und Rehabilitationsphase } \\
\text { G: Videofluoroskopie } \\
\text { E: Aspirationsrisiko }\end{array}$ & $?$ & $76-92$ & $22-67$ & - & - \\
\hline Clave [5] & 2008 & VVST & $\begin{array}{l}\text { P: Patienten mit Schluckschwierigkeiten im Alter, } \\
\text { Z. n. Schlaganfall und anderen neurodegenerati- } \\
\text { ven Erkrankungen } \\
\text { G: Videofluoroskopie } \\
\text { E: Aspirationsrisiko }\end{array}$ & 85 & 84 & 64 & - & - \\
\hline Rofes [25] & 2014 & VVST & $\begin{array}{l}\text { P: Patienten mit Schluckschwierigkeiten im Alter, } \\
\text { Z.n. Schlaganfall und anderen neurodegenerati- } \\
\text { ven Erkrankungen } \\
\text { G: Videofluoroskopie } \\
\text { E: Aspirationsrisiko }\end{array}$ & 120 & 94 & 88 & - & - \\
\hline
\end{tabular}

niels [6], Burke Dysphagia Screening Test (BDST) nach de Pippo [7] und Gottlieb [11] und Standardised Swallowing Assessment (SSA) nach Ellul [10]. Durch Trapl wurde 2007 der Mehrkonsistenzentest GUSS an 50 akuten Schlaganfallpatienten mit einer Sensitivität von $100 \%$ bei einer Spezifität von 50 bis $69 \%$ validiert [28]. Clave hat 2008 den „Volume-Viscosity Swallow Test“ (VVST) mit mehreren Konsistenzen, mit einer Sensitivität von $84 \%$ und Spezifität von $64 \%$, validiert [5]. Hierbei wurden 12 Gesunde (Median 40 Jahre) und 85 Patienten (Median 70 Jahre), davon 40 (Median 75 Jahre) mit v.a. zerebrovaskulären Erkrankungen sowie 24 (Median 60 Jahre) mit neurodegenerativen Erkrankungen, untersucht.
Die oben genannten Ausführungen zeigen, dass die bisher in der Geriatrie verwendeten Dysphagiescreeningverfahren nicht für geriatrische Patienten validiert sind. Lediglich der VVST [5] untersuchte ein teilweise geriatrisches Patientenkollektiv, allerdings mit bereits bestehender Dysphagie.

\section{Praxis des Dysphagiescreenings in der Geriatrie in Deutschland}

Die AG Dysphagie der DGG hat sich 2011 gegründet, mit dem Ziel, den Erfahrungsaustausch zu fördern sowie geeignete Standards zum Management der Dysphagie zu entwickeln. Die Mitglieder der AG sind Ärzte, Logopäden, akademische Sprach- und Schlucktherapeuten und klinische Linguisten, die überwie- gend in Akutkliniken, aber auch in ambulanten Dysphagiezentren tätig sind. Alle haben eine lange praktische Erfahrung in klinischer und apparativer Dysphagiediagnostik.

Durch die Arbeitsgruppe erfolgte 2014 eine Umfrage an deutschen geriatrischen Kliniken zur Praxis der Dysphagiediagnostik. Der Schwerpunkt lag auf der damals vorhandenen apparativen Schluckdiagnostik. Die 55 auswertbaren Rücksendungen zeigten ein inhomogenes Bild. Der Ablauf der Schluckdiagnostik war relativ einheitlich. Es erfolgte zunächst ein bisher nichtstandardisiertes Dysphagiescreening, im Anschluss ein ebenfalls nichteinheitliches Dysphagieassessment durch Logopäden, Sprachtherapeuten bzw. klinische Linguisten. Die Indikationsstellung $\mathrm{zu}$ apparativer 
bzw. bildgebender Diagnostik erfolgte meist geriatrietypisch im Team. In zwei Dritteln der Kliniken existierten klinikinterne Standards zur Dysphagiediagnostik.

Die Umfrage zeigte eine häufige Verwendung des Daniels-Test [6], des GUSS oder auch der Schlucküberprüfung nach Suiter und Leder [14]. Am häufigsten war die Anwendung selbst zusammengestellter und modifizierter Schluckassessments, die häufig mit Unzulänglichkeiten vorhandener Screeninginstrumente begründet wurden. Die Limitationen der vorhandenen Tests lassen sich beispielhaft am Daniels-Test [6] illustrieren, der aufgrund seiner guten Spezifität, Sensitivität und Sicherheit mit schrittweise zunehmenden Volumina des Wasserschlucks in geriatrischen Kliniken in Deutschland verbreitet ist. Aufgrund der Abmessung der definierten Volumina ist der Test aber umständlich und zum Screening wenig alltagstauglich. Der Würgreflex allein als Prädiktor ist relativ unzuverlässig [6]. Auch die anderen validierten Tests erweisen sich aus unterschiedlichen Gründen (aufwendige Zubereitung; notwendige Abmessung von Volumina und Zubereitung von Testkonsistenzen; schlechte Akzeptanz von Testkonsistenzen; notwendige technische Hilfsmittel) als wenig praktikabel in Klinik- und Praxisalltag.

\section{Vorschlag für ein neues Dysphagiescreeninginstrument für geriatrische Patienten}

Basierend auf den Erfahrungen der Mitglieder der AG Dysphagie, der vorliegenden Evidenz sowie der genannten Umfrage wurden Elemente für ein neues Instrument zum Dysphagiescreening zusammengestellt und konsentiert (Mitglieder der Konsensusgruppe • Tab. 3). Für die Zusammenstellung standen Aspekte der Praktikabilität im Mittelpunkt. Voraussetzung war die Durchführbarkeit durch geschultes medizinisches Personal, das keine besondere Qualifikation in Bezug auf die Diagnostik und Therapie von Schluckstörungen braucht.

Der Test besteht aus 3 Elementen (• Tab. 2):

Z Gerontol Geriat 2020 ·53:239-244 https://doi.org/10.1007/s00391-020-01712-7

(c) Der/die Autor(en) 2020

\section{Jäger $\cdot$ U. Thiem $\cdot$ H. Stege}

\section{Entwicklung eines neuen Screeninginstruments zum Screening auf Dysphagie bei geriatrischen Patienten: das Dysphagie Screening-Tool Geriatrie}

\section{Zusammenfassung}

Hintergrund. Die oropharyngeale Dysphagie ist bei geriatrischen Patienten weit verbreitet und fast immer multikausal bedingt. Sie führt zu schwerwiegenden Komplikationen und wird oft unzureichend detektiert. Alle bekannten und etablierten Screeningmaßnahmen fokussieren sich auf Patienten mit neurologischer Grunderkrankung, insbesondere nach Schlaganfall. Material und Methoden. In der Arbeitsgruppe "Dysphagie" der Deutschen Gesellschaft für Geriatrie (DGG), in der Ärzte, Logopäden und Schlucktherapeuten zusammengeschlossenen sind, erfolgte eine selektive Literaturrecherche zum Dysphagiescreening. Basierend auf Ergebnissen der Literaturrecherche und eigenen Erfahrungen wurde durch eine Konsensgruppe ein neues Screeninginstrument für geriatrische Patienten entwickelt und konsentiert. Ergebnisse. Das Screeninginstrument zur Erfassung einer oropharyngealen Dysphagie bei geriatrischen Patienten gliedert sich in 3 Teile: 1. Vigilanz und Sitzposition, 2. Speichelschluck, Zungenbeweglichkeit und Hustenstoß sowie 3. Wasserschlucktest. Das Screeninginstrument kann durch geschultes medizinisches Personal angewendet werden und ist einfach in den Arbeitsalltag zu integrieren.

Diskussion. Das neu entwickelte Screeninginstrument ist speziell für die Anwendung bei geriatrischen Patienten vorgesehen. Es hat Vorteile gegenüber bisher empfohlenen Screeningtests. Bisherige Erfahrungen zeigen eine gute Praktikabilität und Akzeptanz im geriatrischen Team. Weitere Studien müssen die Validität des Instruments prüfen.

\section{Schlüsselwörter}

Älterer Patient · Dysphagie · Schluckstörung · Screening · Geriatrisches Assessment

\section{Development of a new screening instrument for screening of dysphagia in geriatric patients: the dysphagia screening tool geriatrics}

\section{Abstract}

Background. Oropharyngeal dysphagia is widespread in geriatric patients and is nearly always multicausal. It is often insufficiently recognized and leads to severe complications. The available and established screening tools all focus primarily on patients with neurological diseases, usually following a stroke.

Material and methods. The working group on dysphagia of the German Society of Geriatrics (DGG), which is comprised of physicians, speech and language therapists and dysphagia therapists, performed a literature review on dysphagia screening tools. Based on the results of the literature search and own experience, a new screening instrument suitable for geriatric patients was developed and agreed by the consensus group.

Results. The new screening instrument for the detection of oropharyngeal dysphagia in geriatric patients consists of three parts: 1) consciousness and posture control while sitting, 2) ability to swallow saliva and to cough as well as tongue motility and 3 ) the water swallowing test. The screening can be applied by trained medical personnel and can easily be integrated into everyday practice. Conclusion. The new screening tool is especially aimed at geriatric patients. It has advantages compared to previously recommended screening tests. Initial experiences with the instrument show good practicability and acceptance in geriatric teams. Further studies have to test the validity of the instrument.

\section{Keywords}

Aged - Dysphagia - Swallowing disorder . Screening · Comprehensive geriatric assessment 
Tab. 2 Elemente des neuen, konsentierten Dysphagiescreeninginstruments

\begin{tabular}{|l|l|l|}
\hline \multicolumn{1}{|l|}{ Bereich } & \multicolumn{2}{l}{ Bewertung } \\
\hline 1 & $\begin{array}{l}\text { Allgemeinzustand } \\
\text { Ausreichende Vigilanz und Aufmerksamkeit (>15 min) vorhan- } \\
\text { den? }\end{array}$ & Ja \\
\hline $\begin{array}{l}\text { Aufrechte Sitzposition, ggf. mit Unterstützung, möglich? } \\
\text { Orale Inspektion } \\
\text { Beobachtung eines Speichelschlucks möglich? }\end{array}$ & Ja & Nein \\
\hline $\begin{array}{l}\text { Ausreichende Zungenbeweglichkeit? } \\
\text { Effektiver Hustenstoß nach Aufforderung möglich? } \\
\text { Wasserschlucktest }\end{array}$ & \\
\hline $\begin{array}{l}\text { Räuspern oder Husten direkt oder bis zu 1 min nach dem } \\
\text { Schluck? }\end{array}$ & Ja & Nein \\
\hline $\begin{array}{l}\text { Stimmveränderung direkt oder bis zu 1 min nach dem Schluck? } \\
\text { Erläuterungen zur Anwendung und Bewertung: s. Text }\end{array}$ & \\
\hline
\end{tabular}

Tab. 3 Teilnehmer des Konsensverfahrens zur Entwicklung des neuen Dysphagiescreeninginstruments (zentrales Treffen der Arbeitsgruppe „Dysphagie“ der Deutschen Gesellschaft für Geriatrie (DGG; Köln, 23.03.2012))

\begin{tabular}{|c|c|c|c|}
\hline Name & Arbeitsort & Arbeitsstelle & Profession \\
\hline Bange, Christina & Burgbrohl & Geriatrische Rehaklinik & $\begin{array}{l}\text { Logopädin/ } \\
\text { Sprachtherapeutin }\end{array}$ \\
\hline Bergmann, Sonja & Bonn & Gemeinschaftskrankenhaus & $\begin{array}{l}\text { Logopädin/ } \\
\text { Sprachtherapeutin }\end{array}$ \\
\hline Embach, Winfried & Köln & Logopädische Fachpraxis & $\begin{array}{l}\text { Logopäde/ } \\
\text { Sprachtherapeut }\end{array}$ \\
\hline Jäger, Martin & Dinslaken & St. Vinzenz Hospital & Arzt \\
\hline Küsten, Susanne & Bornheim & $\begin{array}{l}\text { Krankenhaus zur Heiligen } \\
\text { Familie }\end{array}$ & $\begin{array}{l}\text { Logopädin/ } \\
\text { Sprachtherapeutin }\end{array}$ \\
\hline Klein, Sascha & Köln & St. Marien Hospital & Arzt \\
\hline Lerch, Michael & Iserlohn & Ev. Krankenhaus & Arzt \\
\hline Maus, Katharina & Bornheim & $\begin{array}{l}\text { Krankenhaus zur Heiligen } \\
\text { Familie }\end{array}$ & $\begin{array}{l}\text { Logopädin/ } \\
\text { Sprachtherapeutin }\end{array}$ \\
\hline Menzel, Joana & Celle & Allgemeines Krankenhaus & $\begin{array}{l}\text { Logopädin/ } \\
\text { Sprachtherapeutin }\end{array}$ \\
\hline Motzko, Manuela & Köln & Kölner Dysphagiezentrum & $\begin{array}{l}\text { Logopädin/ } \\
\text { Sprachtherapeutin }\end{array}$ \\
\hline Orth, Hartwig & \multicolumn{2}{|c|}{ Bergisch-GladbachMarien Krankenhaus } & Arzt \\
\hline Perniss, Markus & Neuruppin & $\begin{array}{l}\text { Ostruppiner Gesundheits- } \\
\text { dienste OGD }\end{array}$ & Logopäde \\
\hline Pitsch, Konstanze & Celle & Allgemeines Krankenhaus & $\begin{array}{l}\text { Logopädin/ } \\
\text { Sprachtherapeutin }\end{array}$ \\
\hline Rittig, Tanja & Dinslaken & St. Vinzenz Hospital & $\begin{array}{l}\text { Logopädin/ } \\
\text { Sprachtherapeutin }\end{array}$ \\
\hline Sickert, Saskia & Dresden & Helios Klinik Schloss Pulsnitz & $\begin{array}{l}\text { Logopädin/ } \\
\text { Sprachtherapeutin }\end{array}$ \\
\hline Stege, Holger & Neuruppin & Ruppiner Kliniken & Arzt \\
\hline $\begin{array}{l}\text { Steiffert, Eva-Ma- } \\
\text { ria }\end{array}$ & Köln & $\begin{array}{l}\text { NTC Neurologisches Therapie- } \\
\text { zentrum }\end{array}$ & $\begin{array}{l}\text { Logopädin/ } \\
\text { Sprachtherapeutin }\end{array}$ \\
\hline Stitz, Bernhard & Hennef & $\begin{array}{l}\text { Kath. Krankenhaus Siebenge- } \\
\text { birge }\end{array}$ & Arzt \\
\hline Wullenkord, Bert & Bornheim & $\begin{array}{l}\text { Krankenhaus zur Heiligen } \\
\text { Familie }\end{array}$ & Arzt \\
\hline
\end{tabular}

1. Beurteilung des Allgemeinzustandes bzw. des Wachheitsgrades

In Anlehnung an den Schottischen Wasserschlucktest [1] und im Wissen, dass sicheres Schlucken nur beim wachen Patienten in aufrechter Sitzposition möglich ist, wurde der Schluckprüfung die Beurteilung von Vigilanz und Sitzfähigkeit vorangestellt. Sollte der Patient nicht ausreichend wach sein bzw. nicht für 15 min aufrecht sitzen können - wobei dazu Hilfen genutzt werden können ist das Screening abzubrechen und die getestete Person als aspirationsgefährdet anzusehen.

\section{Orale Inspektion}

Der zweite Schritt prüft die anatomischen und funktionellen Voraussetzungen des Schluckens. Zunächst erfolgt eine Beurteilung des Speichelschluckens. Der Patient soll aktiv die Zunge bewegen vergleichbar dem Dysphagieassessment nach Logemann [15] - und aktiv husten. Ein effektiver Hustenstoß ist Ausdruck von Muskelkraft und Voraussetzung für suffiziente Reinigungsmechanismen. Sollte eines oder mehrere dieser Manöver nicht möglich sein, ist das Screening als auffällig abzubrechen.

\section{Wasserschlucktest}

Als letzter Teil erfolgt ein Wasserschlucktest. Hierbei werden 2-mal ein Teelöffel (ca. $5 \mathrm{ml}$ ) Wasser nichtangedickt angeboten. Um die Möglichkeit des „Einschluckens" zu nutzen, soll auch bei klinischen Zeichen der Aspiration nach dem ersten Teelöffel ein zweiter angeboten werden. Bei unauffälligem Verlauf sind dann 2 Schluck (je ca. $30 \mathrm{ml}$ ) aus einem Trinkgefäß zu testen. Es sollen keine Schnabelbecher verwendet werden; Hilfestellungen zum Halten des Gefäßes oder Trinkhalme sind erlaubt. Dokumentiert werden Räuspern und Husten sowie eine Stimmveränderung jeweils direkt oder bis zu 1 min nach dem Schluck. Werden klinische Zeichen der Aspiration dokumentiert, ist das Screening als auffällig abzubrechen. Bewusst wird eine Beschränkung auf die wichtigsten Parameter vereinbart und die Zahl der Wasserschlucke 
begrenzt. Auch auf eine Mehrkonsistenzentestung wird verzichtet.

Wurde eine Frage im 1. oder 2. Teil des Screenings mit „nein“ oder im 3. Teil („Wasserschlucktest“) mit ,ja“ beantwortet, wird dies als Zeichen für ein erhöhtes Risiko für eine Dysphagie gewertet und zunächst die Empfehlung zur oralen Nahrungskarenz gegeben. Sollten alle Fragen im 1. und 2. Teil des Screenings mit ,ja“ und im 3. Teil mit „nein“ beantwortet worden sein, kann die orale Nahrungsaufnahme mit der bis dahin empfohlenen Kostform erfolgen.

\section{Diskussion}

Das präsentierte Screeninginstrument ist unseres Erachtens ein pragmatischer Vorschlag für ein einfaches, niederschwellig anzubietendes Dysphagiescreening in der Geriatrie. Das Instrument berücksichtigt vorhandene Nachteile bereits existierender Instrumente. Während der Testphase und Anwendungsbeobachtung durch die Mitglieder der AG Dysphagie zeigten sich eine gute Praktikabilität und Akzeptanz des Instruments. Um eine einheitliche Einweisung in die Anwendung des Instruments zu gewährleisten, hat die AG Dysphagie Schulungsmaterial und eine Präsentation zur Anwendung entwickelt. Mit diesem Material ist eine Einweisung von medizinischem Fachpersonal unkompliziert und mit vertretbarem zeitlichem Aufwand möglich. Das zu schulende Personal benötigt keine besondere Qualifikation in Bezug auf die Diagnostik und die Therapie von Schluckstörungen.

Wir empfehlen die Durchführung des Screenings für bestimmte Patientengruppen im Rahmen der Erhebung des geriatrischen Assessments bzw. im Rahmen der Aufnahme in die geriatrische Klinik oder bei Vorstellung in der Praxis. Unserer Einschätzung nach sollten alle Patienten über 70 Jahre mit folgenden Diagnosen auf eine evtl. vorliegende Dysphagie gescreent werden: FrailtySyndrom, Exsikkose, Sarkopenie, Malnutrition, Gewichtsverlust; akute und chronische neurologische Erkrankungen wie Hirninfarkt, multiple Sklerose, Polyneuropathie und -myopathie; neurodegenerative Erkrankungen wie De- menz, M. Parkinson; COPD, Pneumonie; Delir; Depression, Anpassungsstörung; Polypharmazie; onkologische sowie chirurgisch-orthopädische Erkrankungen in HWS- und Halsbereich. Auch in der ambulanten Praxis und im Pflegeheim kann das Tool erste Hinweise auf das Vorliegen einer Dysphagie liefern und eine entsprechende Diagnostik in Gang setzen.

Bevor eine breite Anwendung des Screenings empfohlen werden kann, muss das Instrument in einer Studie gegenüber einem Goldstandard validiert werden. Daher hat die AG Dysphagie eine diagnostische Studie initiiert, die das Instrument gegen die FEES-Untersuchung testet. Die Ergebnisse dieser Studie werden in Kürze gesondert publiziert.

\section{Fazit für die Praxis}
- Bisher verfügbare Instrumente zum Screening auf oropharyngeale Dys- phagie fokussieren neurologische Erkrankungen.
- Die AG Dysphagie der DGG hat in einem Konsensverfahren ein neues Instrument zum Dysphagiescreening bei geriatrischen Patienten entwi- ckelt und auf der Homepage der DGG zum Download zur Verfügung gestellt.

- Das neue Instrument hat Vorteile gegenüber bisher verfügbaren Screenings und ist durch geschultes medizinisches Personal einfach anzuwenden.

- Vor der Empfehlung für breiteren Einsatz in der Geriatrie müssen Studien das neue Instrument noch validieren.

\section{Korrespondenzadresse}

\section{Dr. Holger Stege}

Klinik für Geriatrie, Ruppiner Kliniken $\mathrm{GmbH}$, Hochschulklinikum der Medizinischen Hochschule Brandenburg (MHB)

Fehrbelliner Straße 38, 16816 Neuruppin, Deutschland

h.stege@ruppiner-kliniken.de

Förderung. Die Arbeit der Arbeitsgruppe Dysphagie wurde mit finanziellen Mitteln der Deutschen Gesellschaft für Geriatrie (DGG) e. V. unterstützt.
Funding. Open Access funding provided by Projekt DEAL.

\section{Einhaltung ethischer Richtlinien}

Interessenkonflikt. M. Jäger, U. Thiem und H. Stege geben an, dass kein Interessenkonflikt besteht.

Für diesen Beitrag wurden von den Autoren keine Studien an Menschen oder Tieren durchgeführt. Für die aufgeführten Studien gelten die jeweils dort angegebenen ethischen Richtlinien.

Open Access Dieser Artikel wird unter der Creative Commons Namensnennung 4.0 International Lizenz veröffentlicht, welche die Nutzung, Vervielfältigung, Bearbeitung, Verbreitung und Wiedergabe in jeglichem Medium und Format erlaubt, sofern Sie den/die ursprünglichen Autor(en) und die Quelle ordnungsgemäß nennen, einen Link zur Creative Commons Lizenz beifügen und angeben, ob Änderungen vorgenommen wurden.

Die in diesem Artikel enthaltenen Bilder und sonstiges Drittmaterial unterliegen ebenfalls der genannten Creative Commons Lizenz, sofern sich aus der Abbildungslegende nichts anderes ergibt. Sofern das betreffende Material nicht unter der genannten Creative Commons Lizenz steht und die betreffende Handlung nicht nach gesetzlichen Vorschriften erlaubt ist, ist für die oben aufgeführten Weiterverwendungen des Materials die Einwilligung des jeweiligen Rechteinhabers einzuholen.

Weitere Details zur Lizenz entnehmen Sie bitte der Lizenzinformation auf http://creativecommons.org/ licenses/by/4.0/deed.de.

\section{Anhang}

\section{Literatur}

1. Anonymous (2010) Management of patients with stroke: identification and management of dysphagia. Scottish Intercollegiate Guidelines Network, Edinburgh

2. Baijens LW, Clave P, Cras P et al (2016) European society for swallowing disorders-European Union geriatric medicine society white paper: oropharyngeal dysphagia as a geriatric syndrome. Clin Interv Aging 11:1403-1428

3. Berzlanovich AM, Fazeny-Dorner B, Waldhoer T et al (2005) Foreign body asphyxia: a preventable cause of death in the elderly. Am J Prev Med 28:65-69

4. Butler SG, Stuart A, Markley L et al (2009) Penetration and aspiration in healthy older adults as assessed during endoscopic evaluation of swallowing. Ann Otol Rhinol Laryngol 118:190-198

5. Clave P, Arreola V, Romea M et al (2008) Accuracy of the volume-viscosity swallow test for clinical screening of oropharyngeal dysphagia and aspiration. Clin Nutr 27:806-815

6. Daniels SK, McAdam CP, Brailey K et al (1997) Clinical assessment of swallowing and prediction of dysphagia severity. Am J Speech Lang Pathol 6:17-24

7. DePippo KL, Holas MA, Reding MJ (1994) The Burke dysphagia screening test: validation of its 
use in patients with stroke. Arch Phys Med Rehabil 75:1284-1286

8. DePippo KL, Holas MA, Reding MJ (1992) Validation of the 3-oz water swallow test for aspiration following stroke. Arch Neurol 49:1259-1261

9. Doggett DL, Tappe KA, Mitchell MD et al (2001) Prevention of pneumonia in elderly stroke patients by systematic diagnosis and treatment of dysphagia: an evidence-based comprehensive analysis of the literature. Dysphagia 16:279-295

10. Ellul J, Barer D (1993) Detection and management of dysphagia in patients with acute stroke. Age Ageing 22:17

11. Gottlieb D, Kipnis M, Sister E et al (1996) Validation of the $50 \mathrm{ml} 3$ drinking test for evaluation of poststroke dysphagia. Disabil Rehabil 18:529-532

12. Hinchey JA, Shephard T, Furie K et al (2005) Formal dysphagia screening protocols prevent pneumonia. Stroke 36:1972-1976

13. Kahrilas PJ (1989) The anatomy and physiology of dysphagia. In: Gelfand DW, Richter JE (Hrsg) Dysphagia: diagnosis and treatment. Igaku-Shoin Medical Pub, New York

14. Leder SB, Suiter DM, Warner HL et al (2011) Initiating safe oral feeding in critically ill intensive care and step-down unit patients based on passing a 3-ounce (90 milliliters) water swallow challenge. JTrauma 70:1203-1207

15. Logemann JA (1983) Evaluation and treatment of swallowing disorders. College-Hill Press, San Diego

16. Martino R, Pron G, Diamant N (2000) Screening for oropharyngeal dysphagia in stroke: insufficient evidence for guidelines. Dysphagia 15:19-30

17. Martino R, Silver F, Teasell R et al (2009) The Toronto bedside swallowing screening test (TORBSST): development and validation of a dysphagia screening tool for patients with stroke. Stroke 40:555-561

18. Muhle P, Wirth R, Glahn J et al (2015) Agerelated changes in swallowing. Physiology and pathophysiology. Nervenarzt 86:440-451

19. Parker C, Power M, Hamdy Set al (2004) Awareness of dysphagia by patients following stroke predicts swallowing performance. Dysphagia 19:28-35

20. Perry L, Love CP (2001) Screening for dysphagia and aspiration in acute stroke: a systematic review. Dysphagia 16:7-18

21. Ramsey DJ, Smithard DG, Kalra L (2003) Early assessments of dysphagia and aspiration risk in acute stroke patients. Stroke 34:1252-1257

22. Rittig T, Jäger M (2002) Strukturen und Methoden des Dysphagiemanagements. Ernährung Medizin 17:180-186

23. Rittig T, Jäger M, Füsgen I (2009) Prävalenz und Bedeutung bei Patienten in geriatrischen Einrichtungen - eine biometrische Multicenter Studie. Eur J Geriatr 2:69-78

24. Rofes L, Arreola V, Almirall J et al (2011) Diagnosis and management of oropharyngeal dysphagia and its nutritional and respiratory complications in the elderly. Gastroenterol Res Pract 2011:818979

25. Rofes L, Arreola V, Mukherjee R et al (2014) Sensitivity and specificity of the eating assessment tool and the volume-viscosity swallow test for clinical evaluation of oropharyngeal dysphagia. Neurogastroenterol Motil 26:1256-1265

26. Smithard DG, O'Neill PA, Park C et al (1998) Can bedside assessment reliably exclude aspiration following acute stroke? Age Ageing 27:99-106

27. Splaingard $M L$, Hutchins $B$, Sulton LD et al (1988) Aspiration in rehabilitation patients: videofluoroscopy vs bedside clinical assessment. Arch Phys Med Rehabil 69:637-640
28. Trapl M, Enderle P, Nowotny $M$ et al (2007) Dysphagia bedside screening for acute-stroke patients: the Gugging swallowing screen. Stroke 38:2948-2952

29. Turley R, Cohen S (2009) Impact of voice and swallowing problems in the elderly. Otolaryngol Head Neck Surg 140:33-36

30. Warnecke T, Dziewas R (2013) Neurogene Dysphagien, Diagnostik und Therapie. Kohlhammer, Stuttgart

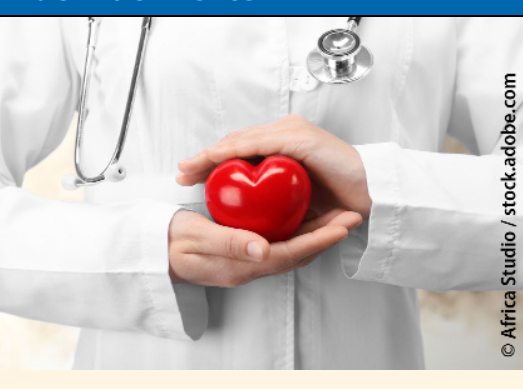

Für starke Frauenherzen

Mehr Frauen als Männer werden wegen einer Herzschwäche im Krankenhaus behandelt und sterben daran. Oft unterschätzen sie die Anzeichen und suchen viel später als Männer ärztliche Hilfe auf. Bei dieser Erkrankung des Herzens reicht die Pumpleistung nicht mehr aus, um den Körper ausreichend mit Sauerstoff zu versorgen. Die häufigsten Gründe sind Koronare Herzkrankheit, Bluthochdruck, Herzrhythmusstörungen, Herzmuskelentzündungen und Herzklappenfehler. Bei Frauen gehen der Herzschwäche häufig ein zu hoher Blutdruck, Erkrankungen der Herzklappen oder auch Diabetes voraus. Symptome wie Atemnot, Müdigkeit oder mögliche Herzrhythmusstörungen sind bei innen initial schwächer ausgeprägt als bei Männern oder werden häufig nicht als Warnsignal interpretiert. Dagegen leiden Frauen häufiger unter möglichen Begleiterscheinungen wie Ängsten oder Depressionen.

Mit einer aktiven, bewussten Lebensweise können Frauen dazu beitragen, Herz und Gefäße gesund zu erhalten und ihr Erkrankungsrisiko zu senken. Unter www.frauengesundheitsportal.de liefert das Frauengesundheitsportal Tipps für mehr Bewegung im Alltag, leicht umsetzbare Ideen für eine herzgesunde Ernährung und wirkungsvolle Strategien zum Abbau von Stress.

Darüber hinaus gibt das Portal einen Überblick zu weiteren Herz-Kreislauf-Erkrankungen wie Bluthochdruck, chronischem Herzversagen oder Herzrhythmusstörungen.

Quelle: Bundeszentrale für gesundheitliche Aufklärung (BZgA), www.bzga.de 